\title{
Eliminated mother to child transmission (MTCT) of HIV and syphilis in Sri Lanka; WHO confirms
}

\author{
Lilani Rajapaksa ${ }^{1}$, G Weerasinghe $^{2}$, Ariyaratne Manathunge ${ }^{3}$, J Elwitigala ${ }^{4}$, Irosha Nilaweera ${ }^{5}$, \\ Kaushalya Kasturiaratchi ${ }^{6}$, Lasanthi Siriwardena ${ }^{7}$, Anuruddha Karunaratn ${ }^{8}$
}

\begin{abstract}
Sri Lanka has been declared as a country that eliminated mother to child transmission of HIV and syphilis by the World Health Organisation (WHO), Geneva on $13^{\text {th }}$ November 2019.

National STD AIDS Control Programme (NSACP) of Sri Lanka

In 1954, commenced a programme for the prevention of congenital syphilis.

In 2002, started the programme for the prevention of mother to child transmission of HIV.

In 2013, these two programmes were combined for the

"Elimination of mother to child transmission (EMTCT) of HIV and syphilis."

Overall target was to achieve and maintain the impact indicators for one year and process indicators for two years and then maintain these achievements to be eligible for the application of the validation process. The programme was designed on a multi-disciplinary, multi-partnered, and multi-level approach with strong links between maternal and child health services and sexually transmitted infections and HIV services in both government and private sectors.
\end{abstract}

By end 2018, the country had achieved the set targets to be eligible to apply for the WHO validation of EMTCT of HIV and syphilis.

In July 2019, the ministry of health made a formal request to the WHO to validate the EMTCT of HIV and syphilis programme. After a thorough assessment of the country situation, the regional validation team recommended certification of Sri Lanka. This was further assessed by the Global validation team at WHO Geneva.

On $13^{\text {th }}$ November 2019, the WHO formally announced that Sri Lanka has eliminated MTCT of HIV and syphilis

Key words: Elimination of Mother to child transmission of HIV and Syphilis, HIV, Syphilis, EMTCT

\footnotetext{
Authors: ${ }^{1}$ Lilani Rajapaksa, MBBS, MSc, MD, Consultant Venereologist, NSACP. ${ }^{1} G$ Weerasinghe, $M D$ USSR, MSC, MD, FRCS (Glasg); Consultant Venereologist, NSACP, ${ }^{1}$ Ariyaratne Manathunge, MBBS, MSC, $M D$, Consultant Venereologist, NSACP, 2J Elwitigala MBBS, MD, Consultant Microbiologist, NSACP, ${ }^{3}$ Irosha Nilaweera, MBBS, MSC, MD, Consultant Community Physician, FHB, ${ }^{3}$ Kaushalya Kasturiaratchi, MBBS, MSC, MD; Consultant Community Physician, FHB ${ }^{4}$ Lasanthi Siriwardena, MBBS, Dip Ven, MD, Acting Consultant Venereologist, NSACP, ${ }^{4}$ Anuruddha Karunaratne; MBBS, Dip Ven, MD; Acting Consultant Venereologist, NSACP,

Corresponding author: ${ }^{1}$ Lilani Rajapaksa, MBBS, MSC, MD; Consultant Venereologist, NSACP
} 


\section{Full Article}

\section{Introduction}

Sri Lanka has been declared as a country that eliminated MTCT of HIV and syphilis by WHO, Geneva in 2019. In September 2015, the UN general assembly adopted the 2030 agenda for sustainable development that includes 17 sustainable development goals (SDGs). Goal 3 is good health and wellbeing. (1) Eradication of Polio, elimination of malaria and measles are major milestones which contributed to a marked reduction in neonatal, infant and child mortality rates in Sri Lanka.

The overall goal for EMTCT of HIV and syphilis is to ensure the prevention of MTCT of HIV and syphilis to a level which is no longer a public health problem. The South-East Asia regional office of the world health organization (WHO SEARO) initially identified Sri Lanka as one of the potential countries in the region to reach the elimination status of MTCT of HIV and syphilis.

1.1. The important reasons to consider Sri Lanka as a potential country to eliminate MTCT of HIV and syphilis.

There are many reasons, but most important is the presence of a well-established free health system with a strong public health sector. The National STD/AIDS Control Programme (NSACP) under the ministry of health has been playing the pivotal role to maintain the current low level of STI and HIV in the country. In 2018 the country's HIV prevalence in the general population was 0.02 with 3,500 estimated PLHIV and syphilis prevalence among STD clinic attendees was 5 per 100,000 adult populations

Sri Lanka, has been able to achieve satisfactory $\mathrm{MCH}$ indicators including MMR and IMR that is markedly lower than the ratios of similar countries. $\mathrm{MCH}$ services in Sri Lanka have a long history, which dates back to the early 20th century. An organized effort to provide $\mathrm{MCH}$ services commenced with the introduction of the Health Unit System in the mid1920s, which was thereafter extended to cover the entire country. The services were further strengthened by establishment of family health bureau (FHB) in 1968. According to the
DHS survey in 2016, $99 \%$ of pregnant women received antenatal care during pregnancy and received trained assistance at delivery.

\subsection{Elimination criteria, indicators, targets in and validation}

Sri Lanka had to achieve the following impact indicators for one year and process indicators for two years to achieve the milestone. But it should be achieved by an adequate "validation standard" national monitoring and surveillance system that can capture process data from both the public and private health sectors, and detect the great majority of cases of MTCT of HIV and syphilis as per the validation readiness criteria.

Indicators and Targets for validation of EMTCT of syphilis and HIV programme

\section{Impact indicators for one year}

- $\quad$ New paediatric HIV infections due to MTCT of $\leq 50$ per 100000 live births

- $\quad$ HIV MTCT rate of $<2 \%$ (non-breastfeeding countries).

- New congenital syphilis of $\leq 50$ per 100000 live births

Process indicators for two years

- Population-level ANC coverage (at least one visit) of $\geq 95 \%$

- Coverage of HIV testing of pregnant women of $\geq 95 \%$

- Antiretroviral therapy (ART) coverage of HIV-positive pregnant women of $\geq 95 \%$

- Coverage of syphilis testing of pregnant women of $\geq 95 \%$

- Treatment coverage of syphilis-positive pregnant women of $\geq 95 \%$

1.3. Criteria for the readiness to apply for the validation

Before applying for validation of EMTCT of HIV and syphilis, Sri Lanka needed to meet the following global minimum criteria.

1 National-level evidence of achievement of the EMTCT validation process indicator targets for two years and achievement of validation impact indicator targets for one year.

2 Evidence that EMTCT of HIV and/or syphilis has been adequately addressed in the lowestperforming subnational administrative units.

3 Existence of an adequate "validation standard" national monitoring and surveillance system that 
can capture process data from both the public and private health sectors, and detect the great majority of cases of MTCT of HIV and/or syphilis

4 Validation criteria must have been met in a manner consistent with basic human rights considerations

According to the WHO, the term "validation" is used when a country has successfully met the criteria for EMTCT of HIV at a specific moment in time. Additionally, validation "implies that countries will also need to maintain ongoing, routine, effective programme interventions and quality surveillance systems to monitor EMTCT of HIV. (2)"

\section{Important landmarks in the path to the elimination of MTCT of HIV and syphilis}

In 2007, the WHO launched the elimination of congenital syphilis programme. Later, this was integrated with EMTCT of HIV and was named as EMTCT of HIV and syphilis. In 2012, the dual EMTCT initiative was initiated. This was presented at the Asia Pacific conference on EMTCT of HIV and syphilis held in September 2015. In response to the request made by the UN agencies from the participating countries, Sri Lanka committed at the meeting, to eliminate MTCT of HIV and syphilis by end 2017.

According to the Global minimum criteria of WHO, a country is eligible to apply for validation of the EMTCT programme if it has achieved the impact indicators for one year and process indicators for 2 years. The plan was to apply for validation based on the achievements during 2017 and 2018.

As per NSACP data, during 2017 and 2018, there were no children reported as having HIV through MTCT and very few children were newly diagnosed with congenital syphilis. (3)

The ministry of health $(\mathrm{MoH})$ had identified the importance of antenatal screening for syphilis as a major component of antenatal services as far back as the 1950s. Even though the prevention programme for MTCT of syphilis has been in place from 1954, the programme has been further strengthened as elimination of congenital syphilis programme in 2009.
A programme called "Prevention of mother to child transmission of HIV" was initiated in early 2002 with the introduction of ART for HIVpositive pregnant mothers. However, until 2012, these services were limited to a few maternity units. In 2012, 99.8\% of pregnant women registered for ANC services were screened for syphilis, of which $60 \%$ were tested in government STD clinics. In the same year, the HIV testing coverage of pregnant women was only $5.6 \%$.

\subsection{Elimination of MTCT of HIV and syphilis programme}

In 2013, the decision was taken to integrate "PMTCT of HIV programme" into the existing elimination of congenital syphilis programme, and the new programme was named as "EMTCT of HIV and syphilis programme". The main objectives were to offer HIV and syphilis testing services for all pregnant women, provide information on prevention of infection among women and to provide treatment services to all pregnant women with HIV or syphilis and to the exposed infants.

The EMTCT programme for HIV and syphilis of Sri Lanka was launched in mid-2013. Highburden districts such as Colombo, Gampaha, Kandy, Matara, Galle, and Hambantota were selected for the initial phase in 2013/2014. Advocacy meetings were conducted in the selected districts to introduce the programme. The $\mathrm{MoH}$ issued a general circular (circular no 02-02/2014) emphasizing the importance of screening pregnant women for HIV and syphilis. A steering committee was formed to guide the programme through regular reviews.

\subsection{Scaling up of EMTCT services from 2013 to 2017}

With the launch of the EMTCT of HIV and syphilis programme in 2013, field-level health care workers providing $\mathrm{MCH}$ services were instructed to offer HIV tests to all pregnant women following pre-test information. Antenatal HIV and VDRL testing were integrated into the existing maternal care package, along with the testing of urine albumin, blood grouping and Rhesus (Rh) factor, blood sugar and haemoglobin. 
Leaflets and posters were developed to increase awareness on the importance of VDRL and HIV testing services. Healthcare workers were trained on the services emphasizing the voluntary testing approach. Until 2012, the practice was to collect $3 \mathrm{cc}$ of blood only for VDRL testing. The amount of blood collected was increased from $3 \mathrm{cc}$ to $5 \mathrm{cc}$ to test for both HIV and syphilis.

Laboratory facilities were improved to cope with the increased demand for HIV testing. Test kits and laboratory equipment such as ELISA machines were procured. International funding agencies, namely UNICEF, World Bank and WHO supported the programme. In 2014, the services were scaled up to cover Northern, North-Central and North-Western provinces. With further scaling up, EMTCT services covered the whole country by end-2016. (4) (Fig.1)

Figure 1-Scaling up of EMTCT services from 2013 to 2016

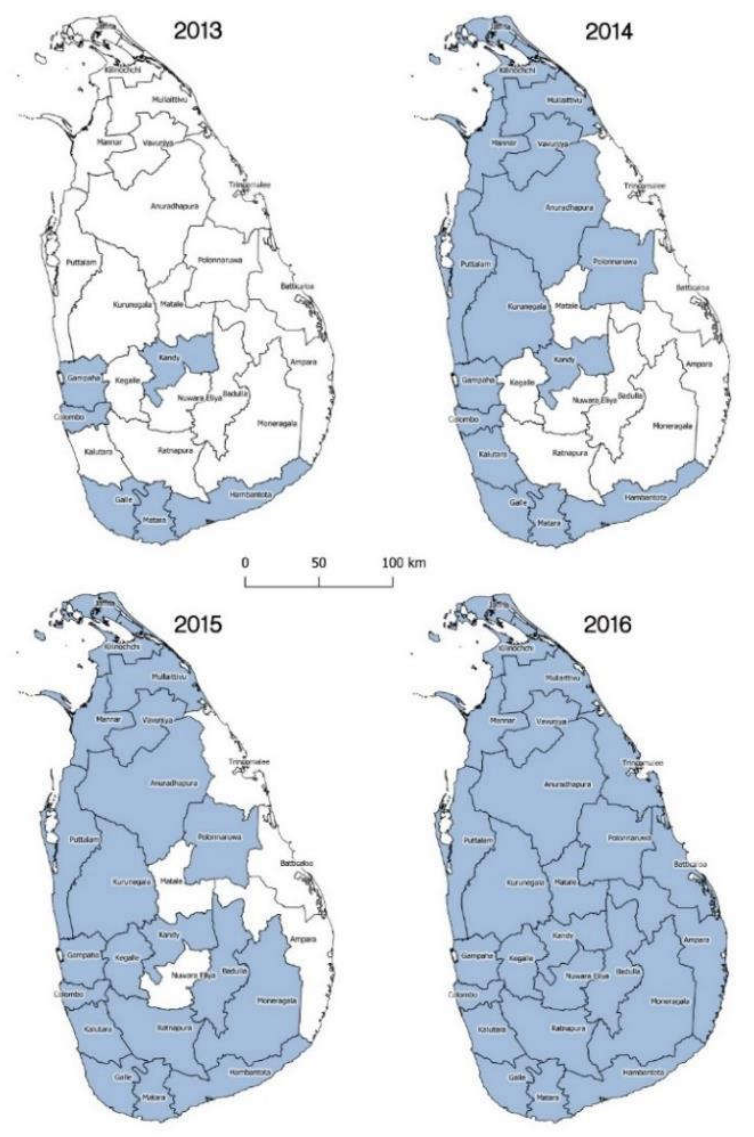

\subsection{Management of pregnant women with HIV}

If the HIV screening test is reactive, the pregnant woman is referred to the STD clinic for confirmatory tests and lifelong HIV care services including EMTCT services.

Pregnant women diagnosed with HIV during pregnancy are managed by the Venereologist in coordination with the relevant $\mathrm{MOH}$, obstetrician and paediatrician.

Pregnant women with HIV are counselled on ART and importance of adherence to treatment, choice of infant feeding, partner disclosure/screening, HIV testing of other children and postpartum family planning by respective consultants. Country adopted treat all policy in 2016 and accordingly pregnant women are started on ART by 14 weeks of POA or as early as possible.

Women presenting close to delivery without a HIV test result are offered a rapid HIV test, and if reactive, EMTCT services are initiated immediately.

\subsection{Diagnosis and management of infants born to mothers with HIV}

Neonatal post-exposure prophylaxis is recommended soon after birth within 4 hours. Infants with standard risk receive syrup NVP for six weeks, HIV DNA/RNA PCR at birth followed by HIV DNA PCR at 8 weeks and 16 weeks(5). In addition to DNA PCR tests, all children receive HIV antibody testing at 9 and 18 months.

BCG vaccination is delayed until the HIV status of the baby is excluded. Other vaccines are given according to the national immunization schedule.

The national nutrition policy of Sri Lanka states "Ensure provision of exclusive breastfeeding for 6 months followed by complementary feeding together with continued breastfeeding for 2 years and beyond". (6)

In Sri Lanka, the most appropriate infant feeding option for an HIV-positive mother depends on many factors including the local situation and the support she is likely to receive. Counselling by a venereologist and a paediatrician is done in collaboration to assist the mother in arriving at a decision. 


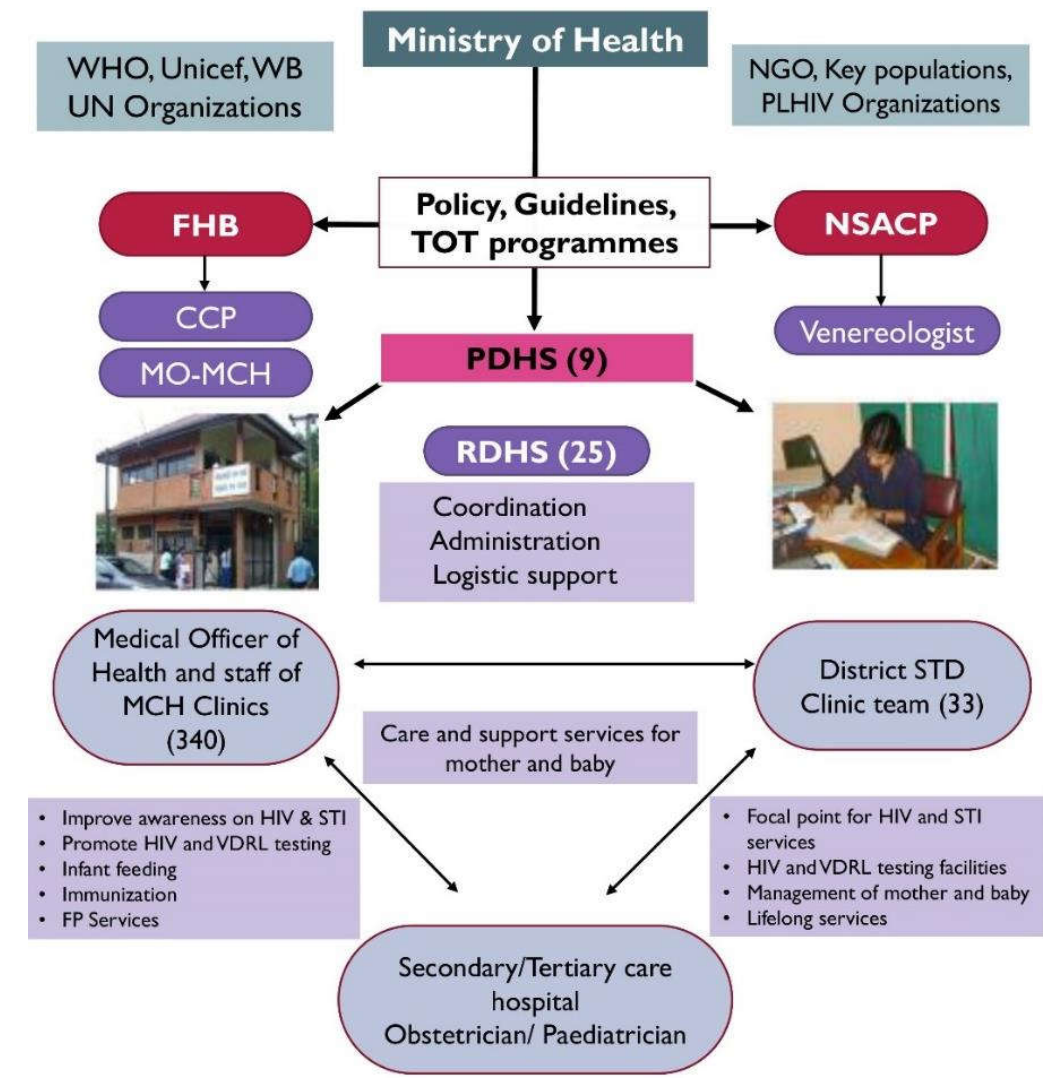

\subsection{Management of pregnant women with} syphilis

All VDRL-positive samples are subjected to confirmatory testing using a treponemal test, TPPA. When TPPA becomes positive, the pregnant woman is referred to the STD clinic for further management. Pregnant women with syphilis are given appropriate treatment preferably with penicillin according to the National guideline. (7) The objective is to complete treatment in early pregnancy. After completion of treatment, the pregnant woman is followed up regularly till delivery, and partner screening and treatment are also completed during this period to prevent reinfection. The obstetrician providing care is informed regarding the plan of management of the mother.

Babies born to pregnant women with syphilis who were adequately treated with penicillin are given a single dose of benzathine penicillin $50000 \mathrm{IU} / \mathrm{kg}$ (body weight) at birth as prophylaxis while other babies are treated as for congenital syphilis. (7)
In the absence of documented evidence of testing for syphilis in pregnancy, syphilis screening is done on admission for delivery and managed accordingly.

\subsection{Reaching Elimination targets}

EMTCT programme planned to achieve several targets by end 2017 based on global minimum indicators for EMTCT of HIV and syphilis. These included increasing HIV and VDRL testing coverage of pregnant women to $>95 \%$, treatment coverage of pregnant women with HIV or syphilis to $>95 \%$ and maintaining an annual rate of congenital syphilis or new paediatric HIV cases at $<50$ per 100,000 live births.

Screening for syphilis among pregnant women in government STD clinics increased from $60 \%$ in 2012 to $99.3 \%$ by the end of 2018 , while HIV testing improved from $5.6 \%$ to $95.9 \%$ during the same period. In 2018, all pregnant women diagnosed with HIV or syphilis received appropriate treatment and babies exposed were followed up till infection excluded. All pregnant women with HIV delivered 
uninfected babies and only a few cases of presumptive congenital syphilis cases were diagnosed.

\subsection{National validation process}

The National Validation Committee (NVC) was formed in 2017 which is chaired by the Deputy Director General (DDG- PHS1). The members of the NVC consisted of administrators, consultants of $\mathrm{MCH}$ and STD services, academic colleges of venereologists, obstetricians and paediatricians, provincial authorities, representatives from NGOs, Key populations (KPs) and People living with HIV(PLHIV). Four working groups were formed under the NVC in March 2017 to work in four main domains of the EMTCT programme, i.e. programmes and services, data management, laboratory services and human rights. They conducted desk reviews, consultative meetings, workshops and surveys when required. The existing standard operating procedures and guidelines were reviewed and updated. In addition, focus group discussions were held with PLHIV groups as well as FSWs to assess the accessibility of reproductive health services. A survey was also conducted among women in postnatal units in 10 major private hospitals in Colombo. (3)

In late 2017, district committees on EMTCT of HIV and syphilis were established to support and monitor activities at the district level.

The National Validation Team (NVT) was constituted in January 2019 under the leadership of the Director General of Health Services(DGHS) to support the submission of the request for validation to WHO Regional Office, South-East Asia by July 2019.

\subsection{Data verification and impact assessment}

A range of methods were used to assess different aspects of the EMTCT programme. The main components of the EMTCT programme were reviewed using WHO validation tools, both quantitative and qualitative methods, drawing on primary and secondary data sources. Routine programme monitoring data, from both the NSACP and FHB were the primary sources of data for impact and process indicators. Denominator values were obtained from the FHB (ANC1) and the Registrar General's Department (registered live births). The estimated number of pregnant women were calculated by adding $10 \%$ to registered live births to compensate for the fetal losses during pregnancy.

\subsection{Data verification}

The Strategic Information Management (SIM) unit of the NSACP has a system for regular collection of data from district STD clinics quarterly. Similarly, the M\&E unit of the FHB collects data from $\mathrm{MOH}$ units directly on $\mathrm{MCH}$ services.

Data were verified in several ways. Supervision of $\mathrm{MCH}$ services (field and hospital) was carried out by expert teams of the FHB to assess data quality. During the district meetings, district (subunit) coverage was analysed based on the defined numerators and denominators. During monitoring and supervision visits to district STD clinics, ANCs and STD clinic laboratories, registers and statistics were reviewed and compared with the data reported to the FHB and the NSACP. Data discrepancies and outliers were investigated and corrected.

For estimates related to women receiving antenatal care and/or delivering in the private sector, the DHS data from 2016 were supplemented by a more recent 2018 survey of private hospitals in Colombo. (3)

\subsection{Laboratory assessment}

The laboratory working group covered four areas including laboratory quality management, quality of tests, quality of testing, and data management.

The National Reference Laboratory of NSACP (NRL) provides technical guidance and reference testing to district STD clinic laboratories which work in a network fashion. The national norms and standards as well as supervision for district STD clinic laboratories are provided by the NRL. Quality assurance and quality control procedures are implemented in all STD laboratories ensuring the quality of testing.

The NRL participates in the external quality assessment (EQA) programme in HIV and syphilis on a regular basis with international PT 
providers and results in the past years have been satisfactory. Additionally, the NRL was working for improving the standards as per ISO-15189 and has applied for certification in 2019. Few assessments of the NRL and the peripheral laboratories were carried out to assess the situation prior to and in mid-term of the laboratory developments to understand the existing capacities. A special internal audit was carried out in NRL by three laboratory auditors (including one external) prior to applying for ISO-15189 accreditation.

The internal quality control, supply chain management, data management and equipment management was looked in to in detail and improved substantially. Laboratory upgrades including infrastructure changes were also carried out.

$\mathrm{CMAI} /$ India supported the quality improvements of the laboratory set up through a project funded by CDC/PEDFAR.

\subsection{Assessment of human rights, gender} equality and civil society engagement

The EMTCT of HIV and syphilis programme is based on strategies and activities consistent with international and national human rights standards. The human rights component was assessed in three areas, namely human rights, gender equality, and civil society and community engagement.

The constitution and laws for the protection of human rights were reviewed. The international conventions which have been adopted in Sri Lanka were extensively reviewed. A desk review was carried out on health sector policies and programmes including NSACP to understand their relevance to human rights, gender equality and civil society engagement. The policies and institutional arrangements relevant to the area of human rights, gender equality and civil society engagement outside the health sector also were reviewed. Judgments given on various relevant incidents also were considered in the desk review.

Interviews were conducted with relevant stakeholders, national HIV and EMTCT programme managers, provincial and regional authorities and health service providers such as consultants, medical officers, nurses and midwives regarding their views on human rights related to HIV. The validation tool checklists were used to assess the human rights component.

\subsection{Achievement of impact and process indicators}

With improvements in detection and management of HIV and syphilis in pregnant women, rates of MTCT for both diseases have fallen well below global elimination targets consistently less than five per 100000 live births (Table 1). The performance of the EMTCT programme currently meets or exceeds 95\% targets for ANC attendance, early screening and treatment for both HIV and syphilis. All pregnant women diagnosed with HIV were started on lifelong antiretroviral treatment (ART) and have shown satisfactory adherence and retention.

Table 1- achievement of Impact and Process indicators

\begin{tabular}{|l|r|r|}
\hline Impact Indicators & $\mathbf{2 0 1 7}$ & $\mathbf{2 0 1 8}$ \\
\hline MTCT rate of HIV & $0 \%$ & $0 \%$ \\
\hline $\begin{array}{l}\text { Annual rate of new paediatric } \\
\text { HIV infections per } 100 \text { 000 live } \\
\text { births }\end{array}$ & 0 & 0 \\
\hline $\begin{array}{l}\text { Annual rate of congenital } \\
\text { syphilis per 100 000 live births }\end{array}$ & 1.5 & 1.5 \\
\hline Process indicators & $97.5 \%$ & 2017 \\
\hline Antenatal care coverage & $95.2 \%$ & $95.9 \%$ \\
\hline $\begin{array}{l}\text { HIV testing coverage of } \\
\text { pregnant women }\end{array}$ & $96.9 \%$ & $99.3 \%$ \\
\hline $\begin{array}{l}\text { Syphilis testing coverage of } \\
\text { pregnant women }\end{array}$ & $100.0 \%$ & $100.0 \%$ \\
\hline $\begin{array}{l}\text { ART coverage of HIV-positive } \\
\text { pregnant women }\end{array}$ & $100.0 \%$ & $97.2 \%$ \\
\hline $\begin{array}{l}\text { Treatment coverage of syphilis- } \\
\text { positive pregnant women }\end{array}$ & \\
\hline
\end{tabular}

Sixteen women living with HIV and 36 women with syphilis delivered during 2018. All pregnant women with HIV received treatment following national protocols, and the newborns remain free of infection. Among pregnant women with syphilis, $97 \%$ received treatment and an annual rate of congenital syphilis was 1.5 per 100000 live births.

Process indicators have improved with $97.5 \%$ in 2017 and $96.4 \%$ in 2018 for coverage of ANC attendance. These figures have been achieved through government services. The screening coverage of pregnant women for HIV was 
95.2\% in 2017 and $95.9 \%$ in 2018 and for syphilis was $96.9 \%$ in 2017 and $99.3 \%$ in 2018 . $(3,8)$.

HIV and syphilis testing coverage among pregnant women increased to $>95 \%$ by end of 2017. (8) By end 2018, the country had achieved the set targets to be eligible to request for validation of EMTCT of HIV and syphilis programme.

\subsection{Visit of the Regional Validation Team (RVT)}

After carefully reviewing the evidence presented by the four working groups, the NVC decided to submit the National validation report to the WHO regional office for the recommendation for validation of EMTCT of HIV and syphilis in Sri Lanka.

The Country report was submitted to the WHO Regional committee in July 2019 and in midSeptember 2019, a seven-member RVT with two observers representing UN agencies and Regional Validation Secretariat visited the country. They assessed the programme through document review, site visits, interviewing key important people and by data triangulation centrally and peripherally. The programme was assessed under the four main domains: Programmes and Services, Laboratory Services, Data Management and Human Rights. The team visited many places including NSACP and FHB and did a thorough assessment in Colombo, Kurunegala and Vavuniya districts, meeting all relevant stakeholders representing STD, MCH, KPs and provincial and district authorities.

The regional validation team submitted their report along with the national validation report to the Global validation Committee (GVAC) in Geneva in October 2019. The report was further assessed by the GVAC through further communication and interviews. Following a strenuous process, the GVAC declared Sri Lanka as a country which has eliminated MTCT of HIV and syphilis in November 2019. The effort taken has been appreciated by the GVAC, WHO Geneva. According to the formal letter sent by WHO, Geneva to the Minister of Health, Nutrition and Indigenous Medicine, the Country validation report submitted by Sri Lanka has been considered of a high standard and the GVAC has requested permission from the country to share the report with other countries planning for EMTCT validation.

The GVAC has identified areas to improve and has given some recommendations which are being implemented currently. It is planned to review the EMTCT programme in November 2021 to assess the sustainability of the success of the programme. It is important to maintain the interest shown by all stakeholders to achieve satisfactory process and impact indicators and to sustain the success in the coming years. The support extended by all stakeholders starting from the $\mathrm{MoH}, \mathrm{FHB}$, provincial authorities, District $\mathrm{MCH}$ services, secondary and tertiary care hospitals as well as NSACP, NRL and district STD clinics has to be commended. Ministry of Health was the main sponsor of the programme and UN funding agencies such as UNICEF, WHO and GFATM supported to further strengthen the programme to achieve the validation certification of EMTCT of HIV and syphilis. Throughout this period the programme worked closely with NGOs, KPs and PLHIV and their contribution at all levels is appreciated.

This programme contributed to the development of the country by assuring child health, reducing maternal illnesses and deaths and by increasing access to services for women in disadvantaged situations. Further, it normalized HIV testing and promoted open discussion on HIV.

\section{References}

1. Nations U. Transforming our world: The 2030 Agenda for sustainable development. 2015;A/RES/70/1.

2. WHO. Global guidence on criteria and processes for validation: EMTCT of HIV and syphilis. 2017.

3. NSACP. NSACP Sri Lanka Annual Report 2018.

4. NSACP. A strategy for elimination of mother to child transmission of HIV and syphilis in Sri Lanka. 2018:6.

5. NSACP. Guidelines for the management of pregnant women with HIV infection. 2016.

6. MoH. National Nutrition policy of Sri Lanka. 2010.

7. NSACP. Guidelines on Management of Pregnant Women with syphilis. 2016.

8. NSACP. NSACP Sri Lanka - Annual Report 2017. 